\title{
Comparative Evaluation of Orthostatic Hypotension in Patients with Diabetic Nephropathy
}

\author{
Gamze Aytaş $^{a}$ Rümeyza Kazancıoğlu ${ }^{b}$ Ömer C. Elçioğlub Meltem Gürsu ${ }^{b}$ \\ A. Serra Artan ${ }^{b}$ Ayşegül Yabacı ${ }^{c}$ Pinar Soysal $^{d}$ Kadir Bilgi $^{a} \quad$ Semra Özçelik $^{e}$ \\ ${ }^{a}$ Faculty of Medicine, Bezmialem Vakif University, Istanbul, Turkey; ${ }^{b}$ Division of Nephrology, Faculty of Medicine, \\ Bezmialem Vakif University, Istanbul, Turkey; ' Department of Biostatistics, Faculty of Medicine, Bezmialem Vakif

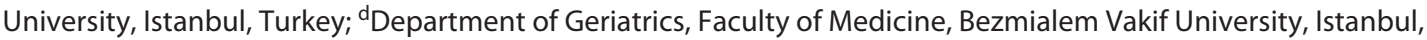

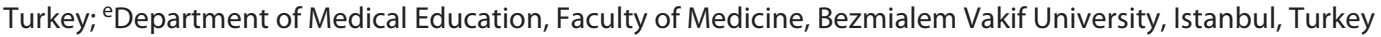

\section{Keywords}

Orthostatic hypotension - Chronic kidney diseases .

Diabetic nephropathy $\cdot$ Blood pressure $\cdot$ Risk factors

\begin{abstract}
Introduction: Orthostatic hypotension $(\mathrm{OH})$ affects $5-20 \%$ of the population. Our study investigates the presence of $\mathrm{OH}$ in diabetic nephropathy (DNP) patients and the factors affecting $\mathrm{OH}$ in comparison with nondiabetic chronic kidney disease (NDCKD) patients. Method: Patients presented to the nephrology clinic, and those who consented were included in the study. DNP was defined by kidney biopsy and/ or clinical criteria. NDCKD patients of the same sex, age, and eGFR were matched to DNP patients. Demographic parameters and medications were obtained from the records. $\mathrm{OH}$ was determined by Mayo clinic criteria. The same researcher used an electronic device to measure blood pressure (BP). All samples were taken and analyzed the same day for biochemical and hematologic parameters and albuminuria. Results: $112(51 \mathrm{~F}, 61 \mathrm{M}$, mean age: $62.56 \pm 9.35$ years) DNP and 94 (40 F, $54 \mathrm{M}$, mean age: $62.23 \pm 10.08$ years) NDCKD patients were included. There was no significant difference between DNP and NDCKD groups in terms of $\mathrm{OH}$ prevalence (70.5 vs. $61.7 \%, p=0.181)$. Male patients had significantly higher $\mathrm{OH}$
\end{abstract}

karger@karger.com www.karger.com/kbr

Karger"

BOPEN ACCESS
(C) 2021 The Author(s)

Published by S. Karger AG, Basel

This is an Open Access article licensed under the Creative Commons Attribution-NonCommercial-4.0 International License (CC BY-NC) (http://www.karger.com/Services/OpenAccessLicense), applicable to the online version of the article only. Usage and distribution for commercial purposes requires written permission. prevalence than female patients ( 74.7 vs. $60.0 \%, p=0.026$ ). There was no significant difference in change in systolic BP between the groups (24.00 [10.00-32.00] $\mathrm{mm} \mathrm{Hg}$ vs. 24.00 [13.75-30.25] $\mathrm{mm} \mathrm{Hg}, p=0.797)$, but the change in diastolic BP was significantly higher in the DNP group (8.00 [2.00$13.00] \mathrm{mm} \mathrm{Hg}$ vs. 6.00 [2.00-9.00] $\mathrm{mm} \mathrm{Hg}, p=0.025)$. In the DNP group, patients with $\mathrm{OH}$ had significantly higher uric acid levels than those without $\mathrm{OH}(7.18 \pm 1.55$ vs. $6.36 \pm 1.65$ $\mathrm{mg} / \mathrm{dL}, p=0.017)$. And, $73.7 \%$ of patients on calcium channel blockers developed $\mathrm{OH}(p=0.015)$, and $\mathrm{OH}$ developed in $80.6 \%$ of 36 patients on alpha-blockers $(p=0.049)$. Conclusion: $\mathrm{OH}$ prevalence is very high in $\mathrm{CKD}$, and etiology of CKD does not have a statistically significant effect on the frequency of $\mathrm{OH}$, despite a difference that could be meaningful clinically. Therefore, patients with CKD are checked for $\mathrm{OH}$, with or without concurrent diabetes mellitus. Evaluation of postural BP changes should be a part of nephrology practice.

(C) 2021 The Author(s)

Published by S. Karger AG, Basel

\section{Introduction}

Diabetes is a leading public health problem with its increasing frequency and a disease with various macro- and microvascular complications. Although it affects one in 
every 11 people between the ages of 20 and 79 years, it still goes undiagnosed for one out of 5 people in the population [1]. Together with the rapid changes in lifestyle, the prevalence of type 2 diabetes is rapidly increasing in all the developed and developing countries [2].

Diabetic nephropathy (DNP) is one of the microvascular complications of diabetes. Its pathology includes glomerulopathy, vasculopathy, and tubulopathy, and it has been reported that $25 \%$ of diabetic patients develop DNP [3]. It is the most common cause of chronic kidney disease (CKD) according to recent studies [4].

Orthostatic hypotension $(\mathrm{OH})$ is defined as a decrease in systolic blood pressure (SBP) of $20 \mathrm{~mm} \mathrm{Hg}$ or a decrease in diastolic blood pressure (DBP) of $10 \mathrm{~mm} \mathrm{Hg}$ within 3 min of standing when compared with blood pressure (BP) from the sitting or supine position [5]. OH, progressing from dizziness to syncope, is a condition that affects $5-20 \%$ of the population; while it is often caused by volume depletion or autonomic reflexes, it can also be caused by medications $[5,6]$. Its frequency varies by comorbidities and age. Studies show that $\mathrm{OH}$ is an important parameter affecting quality of life [7].

Although $\mathrm{OH}$ and DM are a dangerous combination and DNP and $\mathrm{OH}$ are shown to be correlated with and related to each other in the literature [8], there is no study investigating a causal relationship or a pathophysiological link between these pathologies. Therefore, the aim of this study was to investigate and compare the presence and affecting factors of $\mathrm{OH}$ among diabetic and NDCKD patients.

\section{Materials and Methods}

\section{Patients}

In this study, we included patients among those who presented to the Nephrology Outpatient Clinic of the Bezmialem Vakif University Hospital between February and August 2019 and fulfilled the inclusion criteria after obtaining their consent. We have used a frequency match design to eliminate confounding by age, gender, and eGFR; for this purpose, we have formed age-groups for participants at 10-year intervals, except the 18-25 group, and formed eGFR groups based on the GFR staging of CKD ( 5 groups from G1 to G5). We have included every DNP patient who visited the nephrology outpatient clinic and who were previously diagnosed either clinically [9] or with kidney biopsy, and after the DNP patients were included, the nondiabetic CKD (NDCKD) group was created to match the DNP patients in the age, gender, and eGFR distributions.

We have excluded patients whose hemoglobin values were less than $7 \mathrm{mg} / \mathrm{dL}$, who had class 3-4 heart failure according to New York Heart Association classification, who had a stroke in the last 15 days, those with sudden developing tachycardia; dehydration; electrolyte imbalance; acute hemorrhage; sepsis; malignancy; neurological diseases that might lead to $\mathrm{OH}$ such as multiple system atrophy, Parkinson's disease, and spinal cord injury; and those receiving kidney replacement therapies. Additionally, patients who cannot stand up actively by themselves or who were immobile were not included in the study.

\section{Demographic Information}

We have recorded patient's age, gender, additional comorbidities, medications which have been used regularly for the last 30 days at the time of the measurements, and additional relevant medical histories. Missing information was accessed via the hospital's electronic patient files and was recorded.

\section{Orthostatic Hypotension}

$\mathrm{BP}$ measurements were performed with an automatic measurement device (Omron M3 Comfort HEM-7134-E; Omron Healthcare, Co., Ltd., Japan). We ensured that the patients did not take caffeine, smoke, or exercise $30 \mathrm{~min}$ before the measurements. In order to determine $\mathrm{OH}$, systolic and diastolic BPs for all patients were evaluated by the same investigator. The first measurements were taken in the supine position after a rest of at least $5 \mathrm{~min}$, and then patients were asked to rest for 3 more minutes in the supine position. At the end of the 3-min rest, we asked the patients to stand up, and the second measurements were taken while they were standing. The Consensus Committee of the American Autonomic Society and the American Academy of Neurology's definition [5] of $\mathrm{OH}$ which is "a drop of $>20 \mathrm{~mm} \mathrm{Hg}$ in systolic blood pressure (systolic $\mathrm{OH}$ ) and/or a drop $>10 \mathrm{~mm} \mathrm{Hg}$ in diastolic blood pressure (diastolic $\mathrm{OH}$ )" was used to define $\mathrm{OH}$.

\section{Laboratory Findings}

The results of the routine laboratory tests from the nephrology outpatient clinic visits including fasting plasma glucose, urea, uric acid, creatinine, albumin, eGFR, sodium, potassium, phosphorus, calcium, complete blood count (including mean corpuscular volume, mean corpuscular hemoglobin concentration, hemoglobin, and hematocrit), iron, iron-binding capacity, ferritin, HbAlc, spot urine protein/creatinine ratio (ACR) were all obtained and recorded.

\section{Statistical Analysis}

The distribution of the data was examined with the ShapiroWilk test. Normally distributed data are presented as mean \pm standard deviation; nonnormally distributed data are presented as median with interquartile range; and categorical variables are presented as frequency and percentages (\%). To compare the differences among groups, an independent samples $t$ test, one-way ANOVA, and Kruskal-Wallis tests were used for continuous variables, while Pearson $\chi^{2}$ analysis was used for categorical variables. Correlations between parameters were analyzed with Pearson and Spearman correlation tests. Binary logistic regression analyses were performed with diastolic $\mathrm{OH}(\mathrm{DOH})$, systolic $\mathrm{OH}(\mathrm{SOH})$, and $\mathrm{OH}$ as dependent variables to identify and adjust for multiple factors associated with them. Multiple linear regression analyses were performed with changes in SBP and DBP as dependent variables to identify and adjust for multiple factors associated with them. All statistical analyses were done with IBM SPSS statistics 22.0 program (SPSS, Inc., Chicago, IL, USA) and were analyzed and reported at $\alpha=0.05$ significance level, and all $p$ values were two-tailed. 
Table 1. Medications

\begin{tabular}{lccc}
\hline Medication & $\begin{array}{l}\text { NDCKD group } \\
(n=94)\end{array}$ & $\begin{array}{l}\text { DNP group } \\
(n=112)\end{array}$ & $p$ value \\
\hline Insulin, $n(\%)$ & - & $103(92.0)$ & - \\
Diuretics, $n(\%)$ & $32(34.0)$ & $70(62.5)$ & $<0.001^{*}$ \\
ACE/ARB inhibitors, $n(\%)$ & $39(41.5)$ & $59(52.7)$ & 0.109 \\
Ca channel blockers, $n(\%)$ & $46(48.9)$ & $68(60.7)$ & 0.090 \\
Vitamin B supplements, $n(\%)$ & $5(5.3)$ & $13(11.6)$ & 0.111 \\
Allopurinol, $n(\%)$ & $5(5.3)$ & $20(17.9)$ & $0.006^{*}$ \\
Iron supplements, $n(\%)$ & $14(14.9)$ & $17(15.2)$ & 0.955 \\
Neuropathy medications, $n(\%)$ & $1(1.1)$ & $12(10.7)$ & $0.005^{*}$ \\
Alpha-blockers, $n(\%)$ & $10(10.6)$ & $26(23.2)$ & $0.018^{*}$ \\
Beta-blockers, $n(\%)$ & $37(39.4)$ & $58(51.8)$ & 0.075 \\
\hline
\end{tabular}

The Pearson $\chi^{2}$ test was performed for categorical variables. ACE, angiotensin-converting enzyme; ARB, angiotensin receptor blocker; DNP, diabetic nephropathy; NDCKD, nondiabetic chronic kidney disease. * Statistically significant.

Table 2. Biochemical and Hematologic parameters

\begin{tabular}{lccc}
\hline Parameter & NDCKD group $(n=94)$ & DNP group $(n=112)$ & $p$ value \\
\hline Age, years & $62.23 \pm 10.08$ & $62.56 \pm 9.35$ & 0.809 \\
FPG, mmol/L & $5.1[4.6-5.6]$ & $8.1[5.6-12.1]$ & $<0.001^{*}$ \\
Urea, mmol/L & $11.3 \pm 5.6$ & $15.10 \pm 7.44$ & $<0.001^{*}$ \\
Creatinine, $\mu \mathrm{mol} / \mathrm{L}$ & $147.6[115.8-210.3]$ & $153.8[114.92-254.5]$ & 0.576 \\
Uric acid, mmol/L & $0.4 \pm 0.1$ & $0.41 \pm 0.10$ & 0.333 \\
Sodium, mmol/L & $139[138-141]$ & $139[137-140]$ & 0.329 \\
Potassium, mmol/L & $4.54 \pm 0.49$ & $4.7 \pm 0.6$ & $0.022^{*}$ \\
Calcium, mmol/L & $2.4[2.3-2.5]$ & $2.2[2.2-2.4]$ & $<0.001^{*}$ \\
Phosphorus, mmol/L & $1.2[1.0-1.4]$ & $1.2[1.1-1.4]$ & 0.475 \\
Vitamin B12, pmol/L & $236.1[173.4-324.6]$ & $281.47[210.2-383.6]$ & $0.018^{*}$ \\
Albumin, g/L & $42.1 \pm 3.1$ & $39.6 \pm 3.8$ & $<0.001^{*}$ \\
eGFR, mL/min/1.73 m ${ }^{2}$ & $36.00[22.25-52.00]$ & $32.75[20.67-49.59]$ & 0.566 \\
ACR, mg/mmol & $31.67[17.53-126.13]^{\dagger}$ & $141.40[44.40-400.45]^{\ddagger}$ & $<0.001^{*}$ \\
Hemoglobin, g/L & $126.0 \pm 18.3$ & $117.2 \pm 15.5$ & $<0.001^{*}$ \\
MCV, fL & $88.6 \pm 6.5$ & $86.1 \pm 5.9$ & $0.005^{*}$ \\
MCHC, g Hb/L & $326.4[317.8-332.7]$ & $322.7[315.5-328.4]$ & $0.036^{*}$ \\
Iron, $\mu m o l / L$ & $11.5[7.3-14.5]$ & $9.6[7.5-13.6]$ & 0.286 \\
Iron binding, $\mu \mathrm{mol} / \mathrm{L}$ & $35.8 \pm 9.8$ & $43.9 \pm 16.3$ & $0.004^{*}$ \\
Ferritin, $\mu \mathrm{Lg} / \mathrm{L}$ & $149.4[82.1-289.4]^{\dagger}$ & $65.6[36.8-108.9]^{\ddagger}$ & $0.007^{*}$ \\
HbAlc, \% & - & $7.56[6.7-8.7]$ & - \\
\hline
\end{tabular}

The independent samples $t$ test was performed for normally distributed variables. The Mann-Whitney U test was performed for nonnormally distributed variables. ACR, albumin-to-creatinine ratio; DNP, diabetic nephropathy; eGFR, estimated glomerular filtration rate; FPG, fasting plasma glucose; MCV, mean corpuscular volume; MCHC, mean corpuscular hemoglobin concentration; NDCKD, nondiabetic chronic kidney disease. ${ }^{*}$ Statistically significant. ${ }^{\dagger} n=59 .{ }^{\ddagger} n=91$.

\section{Results}

A total of 206 CKD patients participated in this study. 112 of these patients had DNP, and 94 of them had NDCKD. The female-to-male gender ratio of the patients was 1.26 to
1, with 115 female and 91 male patients. The mean age of the patients in our DNP group was $62.56 \pm 9.35$ years, and it was $62.23 \pm 10.08$ years in our NDCKD group.

The biochemical and hematologic parameters of the study groups are presented in Table 1. In the DNP group, 


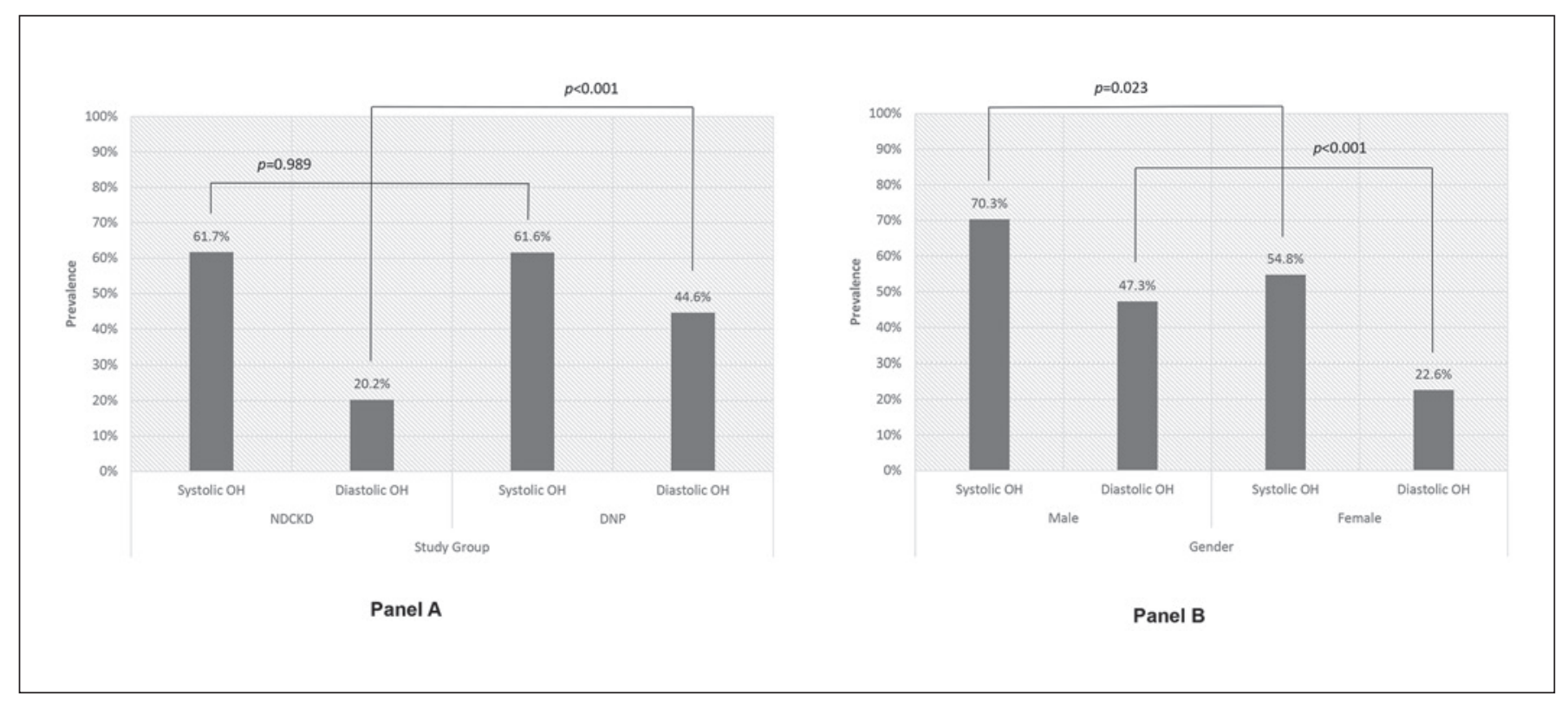

Fig. 1. Orthostatic hypotension prevalence comparison by study groups (A) and gender (B). DNP, diabetic nephropathy; NDCKD, nondiabetic chronic kidney disease. All $p$ values in this figure were calculated with the Pearson $\chi^{2}$ test.

patients with $\mathrm{OH}$ had significantly higher ACRs than those without $\mathrm{OH}(182.69$ [47.51-477.09] $\mathrm{mg} / \mathrm{mmol}$ vs. 105.20 [26.30-220.02] mg/mmol, $p=0.024)$. In the same group, patients with $\mathrm{OH}$ had significantly higher uric acid levels than those without $\mathrm{OH}(0.43 \pm 0.09$ vs. $0.38 \pm 0.10$ $\mathrm{mmol} / \mathrm{L}, p=0.025)$. Serum albumin levels were significantly higher in the NDCKD group than in the DNP group $(42.10 \pm 3.13$ vs. $39.60 \pm 3.72 \mathrm{~g} / \mathrm{L}, p<0.001)$ and in the NDCKD group. Serum albumin levels of patients with $\mathrm{OH}$ were significantly higher than the levels in those without $\mathrm{OH}(43.5$ [41.0-44.0] vs. 42.0 [41.0-42.0] g/L, $p$ $=0.013)$. Urea levels of the DNP group were significantly higher than those in the NDCKD group ( $15.10 \pm 7.44$ vs. $11.30 \pm 5.68 \mathrm{mmol} / \mathrm{L}, p<0.001$ ) (Table 2). We have found that there was a significant correlation between ACR levels and change in DBP $(p=0.043, r=0.159)$. Our results indicate that there is no significant difference in the ACRs between people who have $\mathrm{OH}$ and people who do not have $\mathrm{OH}(p=0.447)$. The most common etiology of CKD among the NDCKD group was hypertension (57.4\%) and the second most common was urological problems (13.8\%), followed by tubulointerstitial nephritis (9.6\%) and polycystic kidney disease (7.4\%). There was no significant relationship between etiologies and $\mathrm{OH}(p=$ 0.152).

The medications of the DNP and NDCKD patients are presented in Table 1. Some medications had a relation- ship with $\mathrm{OH}$, such as $\mathrm{OH}$ prevalence was significantly higher in patients who used calcium channel blockers than that in others (OR: 2.06, 95\% CI: 1.14-3.70, $p=$ 0.015 ), and in patients who used alpha-blockers than others (odds ratio [OR]: $2.37,95 \%$ confidence interval [CI]: $0.98-5.74$, and $p=0.049$ ). In the DNP group, alphablocker use and calcium channel blocker use were associated with significantly higher $\mathrm{OH}$ prevalence (OR: 4.10 95\% CI: $1.13-14.80, p=0.022 / \mathrm{OR}: 2.93$ 95\% CI: $1.26-$ $6.77, p=0.010)$. There was no significant relationship between diuretic use and $\mathrm{OH}$ prevalence in either of the study groups.

There was no significant difference between DNP and NDCKD groups in terms of $\mathrm{OH}$ prevalence (70.5 vs. $61.7 \%, p=0.181)$. When we look at the groups in terms of systolic and diastolic $\mathrm{OH}$ prevalence, the DNP group had significantly higher DOH prevalence (44.6 vs. $20.2 \%$, $p<0.001)$. But there was no significant difference in $\mathrm{SOH}$ prevalence (Fig. 1a). There was no significant difference in change in SBP between the groups (24.00 [10.00-32.00] vs. 24.00 [13.75-30.25] $\mathrm{mm} \mathrm{Hg}, p=0.797$ ), but the change in DBP was significantly higher in the DNP group (8.00 [2.00-13.00] vs. 6.00 [2.00-9.00] mm Hg, $p=0.025)(\mathrm{Ta}-$ ble 3). After binary logistic regression analyses with backward LR elimination method, we have found that study group was an independent factor for DOH (OR: 1.95 95\% CI: $1.38-2.75, p<0.001)$ but not for $\mathrm{OH}$ or $\mathrm{SOH}$ after ad- 
Table 3. BP measurements and $\mathrm{OH}$ prevalence

\begin{tabular}{lccc}
\hline Parameter & NDCKD group $(n=94)$ & DNP group $(n=112)$ & $p$ value \\
\hline SBP in supine position, mm Hg & $142.50[128.75-154.50]$ & $157.00[145.00-170.75]$ & $<0.001^{*}$ \\
SBP in standing position, mm Hg & $121.00[107.75-135.25]$ & $133.50[117.25-154.00]$ & $<0.001^{*}$ \\
Change in SBP, mm Hg & $24.00[13.75-30.25]$ & $24.00[10.00-32.00]$ & 0.797 \\
DBP in supine position, mm Hg & $88.93 \pm 10.89$ & $84.58 \pm 13.49$ & $0.013^{*}$ \\
DBP in standing position, mm Hg & $82.96 \pm 11.59$ & $76.95 \pm 12.39$ & $<0.001^{*}$ \\
Change in DBP, mm Hg & $6.00[2.00-9.00]$ & $8.00[2.00-13.00]$ & $0.025^{*}$ \\
OH prevalence, $n(\%)$ & $58(61.7)$ & $79(70.5)$ & 0.181 \\
SOH prevalence, $n(\%)$ & $58(61.7)$ & $69(61.6)$ & 0.989 \\
DOH prevalence, $n(\%)$ & $19(20.2)$ & $50(44.6)$ & $<0.001^{*}$ \\
\hline
\end{tabular}

The independent samples $t$ test was performed for normally distributed variables. The Mann-Whitney U test was performed for nonnormally distributed variables. The Pearson $\chi^{2}$ test was performed for categorical variables. Change in BP: [BP in the supine position] - [BP in the standing position]. OH, orthostatic hypotension; SBP, systolic blood pressure; DBP, diastolic blood pressure; BP, blood pressure; NDCKD, nondiabetic chronic kidney disease; DNP, diabetic nephropathy. * Statistically significant.

justing for age, sex, eGFR, albumin, hemoglobin levels, alpha-blockers, diuretics, and neuropathy medications. After multiple linear regression analyses with the stepwise elimination method, we have found that the study group was not a factor for change in SBP or DBP after adjusting for age, sex, eGFR, albumin, hemoglobin levels, alpha-blockers, diuretics, and neuropathy medications.

While male patients had significantly higher DBP in the supine position $(89.56 \pm 11.27$ vs. $84.19 \pm 13.01 \mathrm{~mm}$ $\mathrm{Hg}, p=0.002)$, there were no significant differences between the genders in the standing position $(p=0.609)$, and because of that, male patients had a significantly higher change in DBP between positions than female patients (9.0 [5.0-14.0] vs. 5.0 [1.0-9.0] $\mathrm{mm} \mathrm{Hg}, p<0.001)$. In general, male patients had both significantly higher $\mathrm{SOH}$ and $\mathrm{DOH}$ prevalence than female patients $(p=0.023$ and $p<0.001$ ) (Fig. 1b).

\section{Discussion}

Our study demonstrated that $\mathrm{OH}$ is frequent among DNP patients, and actually, it is not more frequent than any other $\mathrm{CKD}$, but reduction in $\mathrm{DBP}$ and the presence of $\mathrm{DOH}$ were more than those in NDCKD. It was also found that in patients with DNP, albuminuria and higher uric acid levels were associated with the development of $\mathrm{OH}$.

Both prolonged life expectancy due to improvement in community health and increase in the trend of etiologic diseases, such as DM and hypertension, cause increased incidence of CKD. In our study, in line with previous studies, the two most important causes of CKD in patients included were hypertension and DM [10]. However, compared to other etiological causes of CKD, DM can make the treatment and management of CKD much more complicated and can increase health expenses [11]. Although two similar groups were created with matching in terms of age, gender, and eGFR in our study, the higher potassium levels and lower levels of calcium, hemoglobin, and iron parameters in the DNP group support that DNP patients were more convoluted.

The dangerous combination of $\mathrm{DM}$ and $\mathrm{OH}$ has been demonstrated in many previous studies, and the combination of $\mathrm{OH}$ and $\mathrm{DM}$ has been found to be associated with numerous adverse clinical outcomes such as increased cardiovascular events and mortality from any cause or decreased quality of life [12-14]. Autonomic denervation causes endothelial dysfunction and reduces neuropeptide responses, volume depletion caused by nephropathy, and osmotic diuresis, which may induce $\mathrm{OH}$. It is not surprising that alpha-blockers and calcium channel blockers, which are known to cause $\mathrm{OH}$, were found to be associated with $\mathrm{OH}$ in our study, as shown previously [6]. In a recently published meta-analysis, risk factors for the development of $\mathrm{OH}$ in those with diabetes mellitus (DM) were investigated, and it was reported that the presence of DNP increased OH 2.37 times, just as in our study [14]. Several studies have shown that one of the factors in the development of mortality and heart failure in patients with $\mathrm{DM}$ and $\mathrm{OH}$ is DNP; this supports our study result indirectly $[13,15]$. It may be that drugs that reduce albu- 
minuria and block RAS are not associated with $\mathrm{OH}$ because they control DNP. One of the interesting results of our study is the correlation between the level of uric acid and $\mathrm{OH}$ in those with DM. Few studies in the literature have investigated orthostatic BP changes with uric acid. In one study, Jiang et al. found no relationship between serum uric acid levels and arterial stiffness causing $\mathrm{OH}$ development [16]. In another study, he found that association of high serum uric acid and increased arterial stiffness was dependent on cardiovascular risk factors in female population [17]. In our study, it is important to find a relationship between uric acid and $\mathrm{OH}$ in patients with $\mathrm{DM}$, which is an important risk factor for the development of cardiovascular disease. Hyperuricemia is one of the numerous cardiovascular risk factors; however, biological effects of uric acid are debatable. Uric acid is a natural antioxidant compound with beneficial characteristics, but preclinical studies have demonstrated that it is also a proinflammatory compound, and an elevated uric acid level has been found to be associated with the risk of myocardial infarction, stroke, and cardiovascular mortality [18]. On the other hand, $\mathrm{OH}$ is already seen at a high rate in CKD patients, and many risk factors in CKD patients, just like DM, can cause arterial stiffness, anemia, impairment of sympathetic nervous system function, and autonomic dysfunction $[19,20]$. In this study, it was shown for the first time that whether the etiology of CKD is DNP or not does not influence the $\mathrm{OH}$ prevalence in these patients.

The limitations of the study are that it is a cross-sectional study and has low sample size, and that we have not recorded the diabetic disease duration of the patients. In addition, the fact that the $\mathrm{BP}$ of the patients was measured only for the 3rd minute while standing but the 1st minute was not evaluated and the fact that the BP measurements of the patients were made only once and that there were no repeated measurements may be other limitations of the study. The strengths of the study are that it is the first study in the literature where the comorbid diseases, drugs, and laboratory parameters are detailed.

It should be noted at this point that in this study, we found the prevalence of $\mathrm{OH}$ in $\mathrm{CKD}$ patients to be much higher than that found in some of the studies in the literature which we believe arises from measurement methods. While the first BP measurements were made while the patients were in a supine position in this study, many of the said studies have made their first measurements while the patients were in seated positions [21]. We should also note that ethnicity of the study population may also be a factor in $\mathrm{OH}$ prevalence, which has been shown by previous studies to be so [22].

Orthostatic Hypotension in Diabetic

Nephropathy Patients
As a result, $\mathrm{OH}$ is common in CKD patients, and contrary to the popular belief, DNP does not increase this frequency. Therefore, patients with CKD, like DM patients, should be evaluated for orthostatic BP at each visit, and potential causes should be reviewed, and necessary precautions should be taken to prevent future $\mathrm{OH}$-related complications.

\section{Statement of Ethics}

This study was approved by the Bezmialem Vakif University Non-Interventional Research Ethics Committee in October 2018 (No: 54022451-050.05.04-). All subjects have given their written informed consent to be included in this study.

\section{Conflict of Interest Statement}

The authors have no conflicts of interest to declare.

\section{Funding Sources}

This study or any research associated with it has received no funding from any organization in the public or the private sector.

\section{Author Contributions}

Gamze Aytaş has designed the study, acquired and interpreted the data, drafted the work, and revised the manuscript. Rümeyza Kazancioglu has contributed to the design of the study, interpreted the data, and contributed significantly to the manuscript. Ömer C. Elçioğlu, Meltem Gürsu, and A. Serra Artan have made contributions to the conception of the study and substantively revised the manuscript. Ayşegül Yabacı has analyzed the data. Pinar Soysal has substantively revised the manuscript. Kadir Bilgi has analyzed and interpreted the data, contributed to the draft of the work, and revised the manuscript. Semra Özçelik has contributed to the manuscript and revised it. All the authors read and approved the final manuscript.

\section{References}

1 Statistics About Diabetes [Internet]. Statistics About Diabetes | ADA. [cited 2020 Jul 29]. Available from: https://www.diabetes.org/resources/statistics/statistics-about-diabetes.

2 Global report on diabetes. Geneva: World Health Organization; 2016.

3 Diabetic nephropathy [Internet]. Mayo Clinic. Mayo Foundation for Medical Education and Research; 2019 [cited 2020 Jul 29]. Available from: https://www.mayoclinic.org/diseases-conditions/diabetic-nephropathy/ symptoms-causes/syc-20354556.

4 Causes of Chronic Kidney Disease [Internet]. National Institute of Diabetes and Digestive and Kidney Diseases. U.S. Department of Health and Human Services; 2016 [cited 2020 Jul 29]. Available from: https://www.niddk. nih.gov/health-information/kidney-disease/ chronic-kidney-disease-ckd/causes. 
5 Consensus statement on the definition of orthostatic hypotension, pure autonomic failure and multiple system atrophy. The Consensus Committee of the American Autonomic Society and the American Academy of Neurology. Clin Auton Res. 1996;6(5):1470.

6 Soysal P, Aydin AE, Koc Okudur S, Isik AT. When should orthostatic blood pressure changes be evaluated in elderly: 1 st, 3 rd or 5 th minute? Arch Gerontol Geriatr. 2016;65:199-203.

7 Gannon J, Claffey P, Laird E, Newman L, Kenny RA, Briggs R. The cross-sectional association between diabetes and orthostatic hypotension in community-dwelling older people. Diabet Med. 2019;37(8):1299-307.

$8 \mathrm{Wu}$ JS, Yang YC, Lu FH, Wu CH, Wang RH, Chang CJ. Population-based Study on the prevalence and risk factors of orthostatic hypotension in subjects with pre-diabetes and diabetes. Diabetes Care. 2009;32(1):69-74.

$9 \mathrm{Yu}$ ASL, Brenner BM, Rector FC. Brenner \& Rectors the kidney. Philadelphia: Elsevier; 2020.

10 Charles C, Ferris AH. Chronic kidney disease. Prim Care. 2020;47(4):585-95.

11 Lim GJ, Liu YL, Low S, Ang K, Tavintharan S, Sum CF, et al. Medical costs associated with severity of chronic kidney disease in type 2 diabetes mellitus in Singapore. Ann Acad Med Singap. 2020 Oct 10;49:731-41.
12 Yoshinari M, Wakisaka M, Nakamura U, Yoshioka M, Uchizono Y, Iwase M. Orthostatic hypertension in patients with type 2 diabetes. Diabetes Care. 2001;24(10):1783-6.

13 Fleg JL, Evans GW, Margolis KL, Barzilay J, Basile JN, Bigger JT, et al. Orthostatic hypotension in the ACCORD (Action to Control Cardiovascular Risk in Diabetes) blood pressure trial. Hypertension. 2016;68(4): 888-95.

14 Zhou Y, Ke SJ, Qiu XP, Liu LB. Prevalence, risk factors, and prognosis of orthostatic hypotension in diabetic patients: a systematic review and meta-analysis. Medicine. 2017; 96(36):e8004.

15 Gaspar L, Kruzliak P, Komornikova A, Celecova Z, Krahulec B, Balaz D, et al. Orthostatic hypotension in diabetic patients-10-year follow-up study. J Diabetes Complications. 2016;30(1):67-71.

16 Jiang Y, Ge JY, Zhang YY, Wang FF, Ji Y, Li HY. The relationship between elevated serum uric acid and arterial stiffness in a healthy population. Vascular. 2020;28(4):494-501.

17 Yuan Y, Huang F, Lin F, Lin M, Zhu P. Association of high serum uric acid and increased arterial stiffness is dependent on cardiovascular risk factors in female population. Horm Metab Res. 2019;51(06):367-74
18 Tuven B, Soysal P, Unutmaz G, Kaya D, Isik AT. Uric acid may be protective against cognitive impairment in older adults, but only in those without cardiovascular risk factors. Exp Gerontol. 2017;89:15-9.

19 Kirkham FA, Rankin P, Parekh N, Holt SG Rajkumar C. Aortic stiffness and central systolic pressure are associated with ambulatory orthostatic BP fall in chronic kidney disease. Nephrol. 2019;33(2):317-24.

20 Liu W, Wang L, Huang X, He W, Song Z, Yang J. Impaired orthostatic blood pressure stabilization and reduced hemoglobin in chronic kidney disease. J Clin Hypertens. 2019;21(9):1317-24.

21 Rouabhi M, Durieux J, Al-Kindi S, Cohen JB, Townsend RR, Rahman M. Orthostatic hypertension and hypotension and outcomes in CKD: the CRIC (Chronic Renal Insufficiency Cohort) Study. Kidney Med. 2021 Mar-Apr; 3(2):206.

22 Strogatz DS, Keenan NL, Barnett EM, Wagner EH. Correlates of postural hypotension in a community sample of elderly blacks and whites. J Am Geriatr Soc. 1991;39(6):562-6. 